\title{
Les dérivés nominaux de noms propres en tant qu'insultes dans le discours médiatique polonais
}

\author{
Nominal derivatives of proper nouns as insults \\ in Polish media discourse
}

\author{
Anna Kochanowska \\ Université Nicolas Copernic \\ aniakochanowska@o2.p1
}

\begin{abstract}
The aim of the article is to study simple nominal derivatives created from the acronym of the Polish political party Prawo i Sprawiedliwość (Law and Justice). In some forms of media discourse, these derivatives function as insults and are among many instruments of hate speech.
\end{abstract}

Keywords: proper nouns, derivatives, insults, hate speech.

\section{INTRODUCTION}

Le titre du présent article met en scène plusieurs phénomènes dont la complexité de nature et celle d'analyse ne peut pas être épuisée par ces quelques pages ; en fait, si la dérivation intéresse surtout les linguistes, l'insulte, pol. obelga, rélève des études sur le langage (Pisarkowa, 1978 ; Grochowski, 1982 ; Kamińska-Szmaj, 2007 ; Grzegorczykowa, 2012), et aussi sur la communication, les médias, la société (cf. Bralczyk, Mosiołek-Kłosińska, 2000, 2001 ; Bukowska, Markowska, 2013, où la notion d'étiquette, pol. etykiet ( $k$ )a, semble servir de terme synonimique à l'insulte). Enfin, les unités de notre analyse ont été précisées en tant que dérivés de noms propres ${ }^{1}$ et le corpus se limite aux commentaires (au sens large) des internautes, animés par la vie politique polonaise, ce qui renvoie sans doute au domaine du discours média-

\footnotetext{
${ }^{1}$ Un nom propre, d'après Jonasson (1994, p. 21) est « toute expression associée dans la mémoire à long terme à un particulier en vertu d'un lien dénominatif conventionnel stable ».
} 
tique largement compris ${ }^{2}$. L'analyse des textes en ligne contenant les dérivés simples formés sur les bases $P i S$ et $P O$ prouve qu'il existe une grande disproportion entre le nombre de dérivés simples créés à partir de l'acronyme $P i S$, et le nombre de dérivés simples formés à partir du sigle $P O$ (nous n'avons trouvé que deux formes : Peów(ka), Powiec/Peowiec).

Tout cela pris en compte, il faut alors préciser l'objet de notre intérêt - nous analysons des dérivés simples, de nature nominale, formés à partir du sigle PiS (du nom propre Prawo i Sprawiedliwość, le parti politique au pouvoir en Pologne) et qui, de par les fonctions qu'ils entretiennent avec le contexte ${ }^{3}$, servent à insulter l'adversaire politique. De cette façon, ces formes non-systémiques constituent un exemple saillant du discours d'hostilité qui parsème le paysage médiatique polonais.

\section{INSULTES ET DISCOURS D'HOSTILITÉ}

Il est évident, comme le rappelle Pisarek (2000, pp. 9-18) qu'une langue nationale se réalise dans les textes transmis dans et par les médias. L'une des caractéristiques du discours médiatique contemporain est sa brutalisation permanente. L'agression dans les médias peut se manifester, selon Satkiewicz (2000, pp. 28-33), de deux façons : soit à travers le choix des moyens langagiers, comme les moyens phoniques, les formes grammaticales et les formes lexicales péjoratives, soit via les effets stylistiques, comme l'ironie, le sarcasme, le ricanement, etc. Les unités de langue que nous analysons, du fait de leur structure morphologique et en interaction avec le contexte, peuvent être qualifiées d'insultes et, par conséquent, font partie de ce que l'on appelle le discours d'hostilité.

\subsection{DÉFINITION D’INSULTE (POL. OBELGA)}

Grzegorczykowa (2012, p. 151) considère que l'insulte se situe quelque part entre réprimande (pol. nagana) et critique (pol. krytyka) et qu'il est alors nécessaire

\footnotetext{
${ }^{2}$ Selon O'Keeffe (2011) « Media discourse refers to interactions that take place through a broadcast platform, whether spoken or written, in which the discourse is oriented to a non-present reader, listener or viewer. Though the discourse is oriented towards these recipients, they very often cannot make instantaneous responses to the producer(s) of the discourse, though increasingly this is changing with the advent of new media technology (...). Crucially, the written or spoken discourse itself is oriented to the readership or listening/viewing audience, respectively. In other words, media discourse is a public, manufactured, on-record, form of interaction. It is not ad hoc or spontaneous (in the same way as casual speaking or writing is); it is neither private nor off the record $»$.

${ }^{3}$ Le contexte comprend l'entourage textuel d'une unité donnée, le contexte paradigmatique et le contexte extralinguistique.

${ }^{4}$ Nous avons choisi le terme français d'insulte en tant que traduction de sa correspondance polonaise obelga - quoiqu'il y ait des doutes parmi les chercheurs français quant à la signification exacte du terme
} 
de définir les conditions de son apparition. Il s'agirait d'un acte de langage qui est intentionnel, et qui consiste à adresser à quelqu'un un jugement négatif (parfois sous forme d'injures), plein de mépris, qui a pour but d'humilier l'interlocuteur. Ainsi, parmi les traits définitoires d'insulte, Grzegorczykowa énumère :

1. Un jugement négatif sur l'interlocuteur (il peut s'agir d'une personne concrète ou d'un groupe, même d'une idéologie),

2. L'intention d'éveiller chez l'interlocuteur le sentiment d'humiliation,

3. Le choix des mots qui véhiculent des émotions négatives (aussi du point de vue axiologique),

4. L'effet perlocutoire - l'interlocuteur se sent insulté.

Toutes les conditions énumérées ci-dessus ne doivent pas être toujours remplies (Grzegorczykowa, op. cit., pp. 158-159), d'autant plus qu'il est souvent difficile de vérifier si un internaute à qui on a adressé des insultes (par ex. un politicien qui lit des commentaires en ligne) se sent réellement humilié et, en conséquence, insulté.

Nous allons défendre l'opinion que les dérivés formés à partir des sigles des noms propres $P i S$ et $P O$, et qui réfèrent aux personnes, comme PiSiaczek, Pełówka, etc., remplissent les conditions d'insulte, c'est-à-dire qu' : 1) ils ont été créés pour exprimer un jugement négatif sur la personne à qui le locuteur s'adresse, ou dont il parle à la troisième personne ${ }^{5}, 2$ ) ils servent à humilier le destinataire à qui on s'adresse ou dont on parle à la troisième personne, ce que prouve l'examen des structures morphologiques employées dans des contextes cités ; et que : 3 ) leur fonction expressive, et parfois aussi poétique sert à véhiculer des émotions négatives envers l'adversaire politique, 4) le destinataire peut se sentir insulté.

Grzegorczykowa (2012, p. 154) précise que le fait d'exprimer un jugement négatif envers un individu (ou un groupe, une religion, etc.) peut prendre la forme d'une injure (pol. wyzwisko), par ex. Ty łajdaku! (fr. Salaud !). En fait, l'analyse de notre matériel prouve qu'il y a des formes qui oscillent entre un surnom et une injure, et c'est l'analyse du contexte qui permet de décider si les conditions d'apparition d'une insulte sont remplies. Grochowski $(1991,2002)$ différencie entre przekleństwo (juron), sémantiquement vide, wulgaryzm (gros mot), qui manifeste les émotions du locuteur d'une façon qui brise le tabou, et enfin wyzwisko (injure) qui sert à manifester les émotions négatives du locuteur envers son interlocuteur. Parmi les dérivés simples que nous analysons, il y a des formes qui révèlent des injures (ne brisent pas

et à sa relation avec les termes apparentés comme injure, invective, blasphème, gros mot, etc. (cf. Lagorgette, Larrivée, 2004), c'est insulte qui semble correspondre le mieux à obelga dans le sens de Grzegorczykowa (2012). Lagorgette, Larrivée (2004, p. 8) proposent de traiter l'insulte comme un acte de langage par lequel on attribue à un allocutaire un groupe nominal véhiculant un contenu axiologique négatif.

${ }^{5}$ Selon Grzegorczykowa (2012, p. 159), l'insulte doit être adressée directement au destinataire ou proférée publiquement. Il semble qu'au moment où un commentaire, une opinion sont publiés en ligne, ils cessent d'être privés et deviennent publics (car n'importe qui peut y avoir accès). 
le tabou, par ex. PiSidto). Celles qui peuvent être qualifiées comme injures et gros mots à la fois (où les émotions négatives rejoignent le tabou) relèvent surtout des contaminations (par ex. PiSdy ${ }^{6}$ ) ou des dérivés complexes (par ex. PiSchuje ${ }^{7}$ ).

\subsection{DISCOURS D’HOSTILITÉ ET NOTION D’ÉTIQUETTE}

Dans les études interdisciplinaires, consacrées au discours politique et au discours public, on rencontre souvent la notion d'étiquette (pol. etykiet(k)a, et/ou les mots du même champ lexical comme etykietować, etykietowanie $)^{8}$. Les limites du présent article ne permettant pas d'exploiter exhaustivement ce sujet vaste, nous allons alors signaler les points communs entre la notion d'étiquette et les traits définitoires de l'insulte, d'autant plus que certains dérivés que nous qualifierions d'insultes comme PiSiaki, PiSiory ${ }^{9}$, PiSjonarze ${ }^{10}$, trouvent leur place dans la liste des étiquettes dans Bukowska, Markowska (2013, pp. 149-170). Markowska (2013, p. 50) définit les étiquettes en tant que quasi-actes de parole qui, contrairement aux noms (pol. nazwy), ne servent pas à identifier l'autre mais à le décrire d'une façon qui impose une interprétation émotionnelle antagoniste. Ainsi, PiSowiec peut fonctionner en tant que nom (nazwa), dont la fonction dominante est d'identifier ${ }^{11}$, mais dans certains contextes la même forme tourne en étiquette, car le locuteur ajoute un autre sens, une autre association méprisante, humiliante, et ceci d'une manière effective (Markowska, 2013, p. 26). Du point de vue grammatical, les étiquettes représentent surtout des groupes nominaux, parmi lesquels on trouve aussi un bon nombre des dérivés créés à partir des noms propres, par ex. Kaczory, kaczyści, Ober-Kaczyński ${ }^{12}$ (Bu-

\footnotetext{
${ }^{6} \mathrm{PiSda}$ réfère au gros mot pizda (le nom du vagin dans le registre familier ou argot, mais aussi une injure pour décrire quelqu'un qui nous déplaît) et à la fois, à la formulation $P i S d a$ (le nom du parti PiS est accompagné de la forme conjuguée du verbe polonais dawać, fr. donner. Cette expression, devenue presque une sorte de phraséologisme, fait penser aux promesses électorales du parti qui se déclarait prêt à donner de l'argent à plusieurs groupes sociaux).

${ }^{7}$ Le dérivé est formé de l'abréviation $P i S$ et du gros mot $c h u j$ (une injure qui sert à nommer une personne et qui renvoie au registre très familier et argotique où chuj veut dire pénis).

${ }^{8}$ Larousse en ligne cite le verbe étiqueter et le définit comme le fait de «classer quelqu'un selon une appartenance idéologique, politique ; cataloguer ».

${ }_{9}^{9}$ PiSiaki, PiSiory font allusion à l'action de pisser et à l'organe mâle de la copulation et de miction (pisiak, pisior).

${ }^{10}$ La forme PiSjonarze fait penser au pluriel misjonarze, fr. missionnaires.

${ }^{11}$ Cela veut dire que le nom n'a aucune fonction expressive ou elle est douteuse. PiSowiec n'est alors autre chose qu'un nom de membre du parti PiS.

${ }^{12}$ Les trois formes renvoient à Jarosław Kaczyński, le leader du partii PiS, personnage influent et controversé. Le nom de famille Kaczyński fait penser à l'appellatif kaczor/kaczka, fr. canard/cane, ce dont profitent les internautes et les opposants de Jarosław Kaczyński pour créer des expressions injurieuses comme kaczory, fr. canards. Kaczyści renvoie au pluriel faszyści, fr. fascistes ; Ober-Kaczyński est une allusion aux grades de la Schutzstaffel.
} 
kowska, Markowska, 2013). En effet, les étiquettes semblent correspondre aux traits définitoires de l'insulte - ce sont les noms qui contiennent des sens additionnels, toujours négatifs, dont la fonction est de stigmatiser, d'agir sur l'interlocuteur et sur l'opinion publique (Markowska, 2013), de discréditer et d'inciter à l'agression et à la haine (Sałkowska, 2013), d'imposer sa vision de l'autre, de l'accuser, de le soupçonner (Głowiński, 2009). Les étiquettes parsèment le discours médiatique et constituent l'un des éléments-clés du discours d'hostilité (Markowska, 2013, p. 20).

La relation entre le terme plus répandu de discours de haine et celui de discours d'hostilité n'est pas toujours claire et facile à cerner. Selon les chercheurs, le discours d'hostilité est une forme de communication particulière qui met l'accent sur l'aversion envers l'adversaire, sur les moyens de le discréditer. Il peut être exprimé directement ou d'une façon indirecte (comme les allusions, les suggestions - Bieliński, 2013, p. 75).

L'attitude émotionnelle négative et la volonté d'insulter l'ennemi politique se manifestent, entre autres, dans le choix conscient que font les internautes d'avoir recours, parmi d'autres moyens langagiers expressifs, à des moyens morphologiques de la création des dérivés (nominaux, verbaux, adjectivaux, etc.). Ces derniers, du fait de leur structure hors contexte ou en coopération avec celui-ci, ne permettent qu'une interprétation expressive négative, hostile ou haineuse, où le référent qui se cache derrière le dérivé nominal devrait se sentir insulté.

\section{LA DÉRIVATION AU SERVICE DU DISCOURS D'HOSTILITÉ}

Les unités étudiées dans le présent travail représentent des lexèmes nominaux, à savoir des dérivés simples, qui ont vu le jour grâce à des opérations morphologiques qui mettent au premier plan les fonctions expressive et poétique en dépit de la fonction représentative. Ils ont été tirés des textes en ligne qui balancent entre l'oralité et les productions écrites et s'approchent du registre familier du langage, en reflétant le discours des situations quotidiennes (Kaproń-Charzyńska, 2014, p. 75).

Prawo i Sprawiedliwość, de même que Platforma Obywatelska, en tant qu'unités de langue, appartiennent sans doute à la catégorie de noms propres ; ils sont lexicalement transparents (ce qui est intentionnel), et dans la plupart des textes médiatiques se trouvent remplacés par les sigles correspondants $P i S$ et $P O$. Dans leur forme intacte, les sigles servent de base à plusieurs opérations de dérivation, de composition, de contamination qui reflètent la richesse et la productivité du système morphologique polonais. Ainsi, plusiers mots nouveaux voient le jour, représentant diverses catégories grammaticales (des verbes comme PiSieć, des adjectifs comme PiSowski, etc.). Comme nous l'avons signalé au début du texte, l'objet de nos observations se limite à un certain nombre de dérivés simples ; nous comprenons le dérivé en tant qu'un mot créé « en ajoutant à un mot existant un élément non autonome ou 
affixe » (Grevisse, Goosse, 2008, p. 162), en plus, un dérivé simple est un mot formé à l'aide d'une seule base à laquelle on ajoute un affixe (dans notre cas, il s'agit de la base $P i S$ - et $P O$ - auxquelles on ajoute un suffixe). L'abondance des structures dérivées, surtout parmi les dérivés composés et les dérivés contaminés (appelés aussi dérivés allusifs - cf. Pałuszyńska, 2006, p. 169 ; Kudra, 2001, p. 128) demanderait de traiter toute unité à part. C'est dans la catégorie des dérivés composés et des contaminations que l'on constate la présence directe (par ex. POjeby, POsrańce ${ }^{13}$ ) ou indirecte (allusive, par ex. PiSdy, PiSchuje) des gros mots car la dérivation simple n'implique autre radical que $P i S$ ou $P O$. En plus, l'acronyme $P i S$ crée quatre-vingt-dix pour cent des dérivés simples, la base consonantique et la capacité à connoter étant probablement responsables de cette disproportion ${ }^{14}$. Nous admettons que ce fait influe sur nos analyses ; en fait, les dérivés simples que font l'objet de la présente étude ont été créés surtout à partir de l'acronyme $P i S, P O$ ne participe qu'à la formation du dérivé simple Peów(k)a.

\subsection{LES FORMES OCCASIONNELLES (POL. OKAZJONALIZMY)}

L'observation des dérivés simples en ligne contenant les sigles $P i S$ et $P O$ pose la question du statut proprial des dérivés. En fait, si la grammaire polonaise exige que tout nom propre s'écrive avec une majuscule, les internautes ont recours à différentes écritures des acronymes, d'ailleurs facilement reconnaissables grâce au contexte. Pour plus de clarté, nous citons nos dérivés avec la forme correcte (majuscule) des acronymes (PiSiulka, POwiec) mais, en réalité, nous observons tout un éventail de combinaison majuscule-miniscule, par ex. Pisuś, pisulec... Bien sûr, la communication en ligne fait souvent fi des règles de grammaire ; il est alors difficile d'évaluer l'intentionnalité de l'emploi de telle ou telle orthographe. Quoi qu'il en soit, aucun dérivé que nous étudions n'est répertorié dans les dictionnaires traditionnels de la langue polonaise. Parmi les dictionnaires en ligne, seules les formes PiSowiec et POwiec (et leurs variantes graphiques) trouvent leurs entrées, d'ailleurs pas unanimes ${ }^{15}$. Il en résulte que nous avons affaire à des formes occasionnelles, nonsystémiques, textuels qui sont créées ad hoc par le locuteur (Nagórko, 2003, p. 175).

\footnotetext{
${ }^{13}$ Les appellatifs pojeby et posrańce sont fortement injurieux, employés envers les personnes que l'on croit stupides, déséquilibrées, bizzarres, etc.

${ }^{14}$ La base $P O$ est plus apte à apparaître dans diverses contaminations où, par un jeu de langue qui consiste à reconnaître l'acronyme dans la structure du mot (POwiedzieć, POstępowy, POpapraniec, etc.), les internautes ajoutent le sens expressifs aux unités de langue, qui, souvent, ne sont pas expressifs du point de vue du système.

${ }^{15} C f$. Dobrysłownik.pl. Retrieved from https://dobryslownik.pl ; Słownik SJP.PL. Retrieved from https://sjp.pl ; Stownik języka polskiego PWN. Retrieved from https://sj.pwn.pl (qui traite les formes dans la section orthographique).
} 
Ces formes brisent souvent les règles morphologiques du système et deviennent expressives.

Il semble que dans le cas des formes occasionnelles comme PiSuś (nom masculin expressif qui réfère au membre du parti $P i S$ ), Pisiarka (nom féminin expressif qui réfère au membre du parti $P i S$ ), etc., les internautes créent des dérivés onomastiques occasionels que nous devons considérer en tant qu'appellatifs car ils appartiennent plutot à la catégorie de noms insultants, d'inujres (pol. wyzwisko) qu'à la catégorie des surnoms (pol. przezwisko) ${ }^{16}$. Contrairement aux dérivés systémiques (comme judasz, casanova), les formes occasionnelles textuelles pourraient avoir pour but d'insulter, de discréditer l'adversaire politique, c'est pourquoi elles imposent une interprétation péjorative du référent et véhiculent des émotions négatives (Kamińska-Szmaj, 2006, pp. 108-109).

\subsection{LA DÉRIVATION EXPRESSIVE À L'AIDE DES MOYENS SYSTÉMIQUES}

Les formes que nous analysons - PiSol, PiSiuch, PiSiara, PiSiarka, PiSówa, PiSówka, PiSor, PiSulec, PiSisko, PiSidło, PiSiak, PiSuś, PiSiaczek, PiSulek, PiSiulka, PiSiątko, constituent des dérivés expressifs ; sans entrer dans le détail de la littérature sur l'expressivité, nous admettons qu'il y a des moyens langagiers dont la fonction primaire est de véhiculer une/des émotion(s), de maniferster les sentiments et la personnalité du destinateur ( $c f$. Grabias, 1981, p. 17). Parmi eux, on classifie des suffixes expressifs ${ }^{17}$ qui contribuent à la création des dérivés expressifs. La seule source de l'expressivité de nos dérivés vient de la fonction expressive des suffixes.

$\mathrm{Si}$ la fonction expressive sert à manifester des émotions positives ou négatives via l'emploi des suffixes expressifs systémiques, la fonction poétique consiste à créer des structures qui intentionnellement se placent hors norme car les morphèmes ou les dérivés ont été placés dans un contexte atypique (par ex. PiSduś, à travers la contamination, met ensemble la base péjorative pizd(a) et le suffixe mélioratif -uś, ce qui viole les règles de la collocation des morphèmes, $c f$. KaprońCharzyńska, 2014, pp. 168-187; Pisior peut apparaître dans un contexte qui le fait intepréter non seulement comme un synonyme familier du lexème penis mais comme un nom allusif du membre du parti PiS).

\footnotetext{
${ }^{16}$ Le surnom sert surtout à identifier, une injure à exprimer l'attitude émotionnelle du destinateur. On peut trouver des exemples des dérivés simples qui restent dans la catégorie de noms propres mais, dans la grande majorité des cas, il s'agit de bases constituées par des prénoms (par ex. Donaldinio, allusion à Donald Tusk) ou des noms de famille (par ex. Kaczor, Niesioł, Krzak, allusions à Jarosław Kaczyński, Stefan Niesiołowski, Marian Krzaklewski, cf. Pałuszyńska, 2006).

${ }^{17}$ Les suffixes expressifs de la langue polonaise ont été largement étudiées, la bibliographie complète a été présentée par Burkacka (2012, pp. 129-153) et Kaproń-Charzyńska (2014).
} 
Notre liste contient les dérivés simples qui, dans un contexte donné, véhiculent une expressivité : 1) conforme à la fonction expressive potentielle des suffixes : PiSol, PiSiuch, PiSiara, PiSówa, PiSor, PiSulec, PiSisko, PiSidło, PiSiak, 2) non conforme à la fonction expressive potentielle des suffixes : PiSuś, PiSiaczek, PiSiulek, PiSiulka, PiSiątko.

Dans le premier groupe, se trouvent les dérivés simples créés au moyen des suffixes expressifs péjoratifs. Leur emploi sert à transmettre des émotions négatives ce qui correspond à la fonction expressive potentielle des suffixes ${ }^{18}$. Dans tous les cas cités ci-dessus, il s'agit de la combinaison de la base $P i S$ - ou $P O$ - (soit de sa variante peo-) avec les suffixes expressifs péjoratifs (et parfois aussi augmentatifs) ${ }^{19}$ invariants (a-d) ou contextules (e-j) :

a. PiSol : „PISol, a gada do rzeczy...” (http://supernowosci24.pl/co-z-budowacentrum-wystawienniczo-kongresowego-w-jasionce/) ${ }^{20}$;

b. PiSiuch : „Wow, tyle niewygodnej prawdy w 1 komentarzu i jeszcze żaden pisiuch nie zaminusowat?" (http://www.wiocha.pl/komentarze_uzytkownika, 217838,308) ${ }^{21}$;

c. PiSiara: „Kolejna PISiara po Hardeju, który mleczarnie doprowadził do bankructwa, brawo sokołowianie dalej głosujcie na nierobów z PIS-u", (http://www.wiescisokolowskie.pl/zostala-na-bruku,08I8GHWAWpES3ZOof HK1TCB9bO936V2DSA576wzs325N946TZ1.html) ${ }^{22}$;

d. PiSówa, Peówa, Pełówa (avec l'élément $t$ qui sert à joindre la base avec le suffixe, pol. konektyw) : „Straszna pisówa wyrzucona z Wiadomości” (http://stoje-z-boku-i-patrze.salon24.pl/244478,straszna-pisowa-wyrzucona-zwiadomosci) ${ }^{23}$; ,Dyżurna pełówa już pluje jadem. Prokuratura jest pisowska według kłamczuchy platformianej. Znawczyni polityki a nie wie ile ma lat raz twierdzi że ma 29 lat a raz że jest po 60tce. Emerytowana ubeczko co upublicznią pytam?..” (http://wiadomosci.onet.pl/forum/stenogramy-z-kabinypilotow-nie-beda-upublicznione,299257,20,czytaj-popularne.html ${ }^{24}$;

\footnotetext{
${ }^{18}$ Pour la répartition des suffixes, nous avons recours au classement proposé par KaprońCharzyńska (2014, pp. 128-130) et Grzegorczykowa, Puzynina (1979).

${ }^{19}$ Nous ne prenons pas en compte la fréquence de l'emlpoi des formes citées (en fait, il y a des dérivés fréquents commme PiSior et rares comme PiSur). Telles études demanderaient un traitement à part, qui aurait recours aux études sur la fréquence de la collocation des suffixes avec la base substantivale (cf. par ex. Burkacka, 2012).

${ }^{20} \mathrm{fr}$. «<PiSol>, et pourtant il sait parler avec sens ». [trad. de l'auteure]

${ }^{21} \mathrm{fr}$. « Wow, autant de vérité incommode dans 1 commentaire et pourtant, aucun <pisiuch $>$ ne l'a pas encore éreinté ? ». [trad. de l'auteure]

${ }^{22}$ fr. «Une autre $<$ PiSiara $>$, après M. Hardej qui a mené à la faillite nos laiteries, bravo, les habitants de Sokołów, continuez à voter pour les fainéants de PiS ». [trad. de l'auteure]

${ }^{23}$ fr. «L'horrible <pisówa> a été viré de Wiadomości [journal de la télé publique polonaise] ». [trad. de l'auteure]

${ }^{24} \mathrm{fr}$. « $<$ Pelówa $>$ de service commence déjà à cracher son venin. Le parquet est dans les mains de PiS, selon la menteuse de PO. Elle se dit expert en politique mais ne sait pas quel âge elle a (...). Une
} 
e. PiSor, PiSior : „Platfusy: Chcecie żeby pisory posłały nas do mamra? Lud: TAK!” (https://wzzw.wordpress.com/2013/05/31/platfusy-chcecie-zeby-pisoryposlaly-nas-do-mamra-lud-tak- $\% \mathrm{E} 2 \% 98 \% 9 \mathrm{~A} /)^{25}$;

f. PiSulec : „, (..) Głupi naród wierzył we wszystko co mu kurdupel i spółka obiecywali a im szło jedynie o, jak to ładnie powiedział Pan Prezydent'Po 8 latach zwrócono im doją [sic!] ojczyznę. Najgłupszy pisulec na stanowisku będzie lepszy od mądrych inaczej czyli drugiego sortu” (http://m.interia. pl/komentarze,2315111,4211,strona, 7$)^{26}$;

g. PiSisko : „Alex_Disease twierdzi, że PiS znowu coś zawłaszcza. Tym razem biedne pisisko rzuciło się na ŻN. No cóż - przykro mi, że tylko PiS czuje się spadkobiercą idei Żołnierzy Niezłomnych..." (http://zamkinapiasku.salon24. pl/490632,byc-polakiem-nie-jest-latwo-w-polsce) $)^{27}$;

h. PiSidto : „A pisidło swoje! Nikt cię zakało nie słucha bo dla wielu pis to wstyd. Przeglądając twoje posty przychylam się do tej powszechnej opinii. Nigdy więcej pisich rządów" (http://forum.echodnia.eu/kaminski-z-wyrokiema-co-z-afera-dudy-t290212/) ${ }^{28}$;

i. PiSiak: „PiSiaki, TV Trwam i inne wieści z zakrystii” (http://lekkoduch.blog.onet.pl/2014/11/05/pisiaki-tv-trwam-i-inne-wiesci-z-zakrystii/) ${ }^{29}$.

Le deuxième groupe comprend les dérivés qui ne se trouvent pas dans les contextes typiques, naturels pour l'expressivité qu'ils véhiculent, c'est-à-dire qu'ils sont mis dans les contextes qui s'opposent à l'expressivité manifestée à travers leurs suffixes expressifs. En général, il peut s'agir de la situation où : 1) les dérivés avec les suffixes mélioratifs sont employés dans les contextes qui imposent une inter-

salariée de l'Office de Sécurité communiste, à la retraite, qu'est-ce qu'ils vont rendre public, je te le demande, hein ? ». [trad. de l'auteure]

${ }^{25} \mathrm{fr}$. «Les partisans de $P O$ [mot familier intraduisible en français] : Vous voulez que les <pisiory> vous jettent en taule ? Le peuple : OUI ! ». [trad. de l'auteure]

${ }^{26} \mathrm{fr}$. «(...) Le peuple sot et crédule croyait à tout ce que lui promettait le nabot [l'allusion à Jarosław Kaczyński et à sa petite taille] et sa compagnie et eux, ils voulaient seulement, comme l'a joliment dit monsieur le président, qu'on leur rende la patrie qu'ils vont écorcher. Le plus stupide $<$ pisulec $>$ dans son poste sera meilleur que le plus intelligent parmi les intelligents autrement, c'est-à-dire les gens de la seconde catégorie [allusion aux mots de Jarosław Kaczyński dans lesquels il a appelé ses opposants politiques " des gens de seconde catégorie" ». [trad. de l'auteure]

${ }^{27}$ fr. « Alex Disease [un bloggeur] affirme que PiS s'empare de nouveau de quelque chose. Cette fois le pauvre $<$ pisisko $>$ s'est jeté sur les soldats maudits [ceux qui n'ont pas cédé au pouvoir communiste après la fin de la deuxième guerre mondiale]. Ben, c'est marrant qu'il n'y a que PiS qui se sent l'héritier de l'idée de soldats maudits [Les soldats dits maudits du réseau anti-communiste clandestin] ». [trad. de l'auteure]

${ }^{28}$ fr. « Et <pisidło> continue sa merde ! Personne ne t'écoute, espèce d'empoisonneur parce que pour nombreux pis est une honte. Quand je lis tes courriers, je partage l'opinion commune. Plus jamais de $P i S$ au pouvoir ». [trad. de l'auteure]

${ }^{29}$ fr. « $<$ Pisiaki $>$, TV Trwam [la station télévisée catholique, liée au parti PiS] et d'autres infos de la sacristie ». [trad. de l'auteure] 
prétation péjorative (par ex. ironique, Kaproń-Charzyńska 2014, p. 151), 2) les dérivés avec les suffixes péjoratifs sont employés dans les contextes qui imposent une interprétation positive (méliorative, $c f$. Kaproń-Charzyńska 2014, pp. 157-159). L'observation de nos dérivés prouve que dans un discours d'hostilité, bâti sur les insultes, on a toujours affaire à une interprétation négative du dérivé, c'est-à-dire que les internautes ont recours à des dérivés expressifs potentiellement (systémiquement) positifs pour créer un discours négatif (ce qui reflète la fonction poétique des dérivés). Ainsi, dans tous les exemples cités dessus, les suffixes mélioratifs (parfois aussi diminutifs) qui normalement, dans les contextes typiques de l'usage, créent les dérivés expressifs mélioratifs, modifient leur fonction - ils servent à manifester le mépris, l'ironie, le ricanement, le sarcasme, etc. (tous les suffixes expressifs sont invariants) :

j. PiSuś : „...Towarzysze partyjni, atakując zajadle szydzących z PiSusia, podgrzali tylko atmosferę i uruchomili w internecie kolejną lawinę jeszcze bardziej kąśliwych i niewybrednych dowcipów..." (http://soc.culture.polish.nar kive.com/Plwf1NS5/andrzej-dupa) $)^{30}$;

k. PiSiaczek :,2 straconych lat dla Polski gdyby rządził pisiaczek” (http://www. bankier.pl/forum/temat_re-12-straconych-lat-dla-polski-gdyby-rzadzil-pisiaczek, 12972808.html) ${ }^{31}$;

1. PiSiulek: „I PiSiulek Karski Karol, wiceminister od spraw zagranicznych u Fotygi, się popisał: z wysokości swojej „kompetencji” starał się poniżyć Tuska [sic!]" (http://pl.soc.polityka.narkive.com/HMF1nHFY/pisiulki-dwieciemna-i-jasna-i-pisiulek-jeden) ${ }^{32}$;

m. PiSiulka : „PiSiulki dwie, ciemna i jasna / oburzone są tym, że szefem jednej z komisji został Marek Borowski, a nie PiSiulek Adam Lipiński...” (http://pl. soc.polityka.narkive.com/HMF1nHFY/pisiulki-dwie-ciemna-i-jasna-i-pisiulekjeden $)^{33}$;

n. PiSiątko : „Następne rozkosznie naiwne pisiątko, zagubione w polityce jak we mgle" (https://www.wykop.pl/wpis/23976337/chyba-nielada-poploch-wzaciszu-biur-mlodziezowek-/) $)^{34}$.

${ }^{30}$ fr. «... Les camarades du parti [allusion au communisme], qui ont dirigé leurs attaques contre ceux qui avaient ricané de $<$ PiSuś $>$, n'ont fait autre chose que de mettre de l'huile sur le feu et de provoquer une autre avalanche des blagues incisives $»$. [trad. de l'auteure]

${ }^{31}$ fr. «Deux années gaspillées en Pologne si un < pisiaczek> arrivait au pouvoir ». [trad. de l'auteure]

${ }^{32}$ fr. « Le $<$ PiSiulek $>$ Karski Karol, le vice-ministre des affaires étrangères chez Mme Fotyga, vend sa salade : de sa „,compétence” très élevée, il essaie d'humilier Tusk ». [trad. de l'auteure]

${ }^{33}$ fr. « Les deux $<$ Pisiulki $>$, la blonde et la brune, s'indignent de ce que Marek Borowski est à la tête de l'une des commissions, pas le $<$ Pisiulek $>$ Adam Lipiński ». [trad. de l'auteure]

${ }^{34}$ fr. «Un autre < pisiątko > délicieusement naïve, perdue dans les méandres de la politique comme dans un brouillard». [trad. de l'auteure] 
L'expressivité de tous les dérivés nominaux inventoriés ci-dessus est due aux suffixes: soit les internautes ont recours aux suffixes péjoratifs pour créer des dérivés péjoratifs, soit ils profitent de leur créativité pour sortir de la norme et jouer avec une interprétation péjorative des dérivés systémiquement mélioratifs. Les émotions véhiculées dans les deux cas ne sont pas les mêmes; si nous voulions décrire nos dérivés à l'aide des qualificateurs pragmatiques ${ }^{35}$, le premier groupe des dérivés pourrait être accompagné des qualificateurs émotifs comme méprisant (pol. lekceważące) - 'l'émetteur n'exprime pas son respect', stigmatisant (pol. piętnujące) 'l'émetteur ressent une grande aversion', dédaigneux (pol. pogardliwe) - 'l'émetteur ressent une grande aversion orgueilleuse'. Aux qualificateurs déjà mentionnés, le deuxième groupe ajoute les qualificateurs poétiques (Kaproń-Charzyńska 2014, p. 122) comme ironique (pol. ironiczne) - 'l'émetteur renverse son jugement, il dit autre chose qu'il ne pense, il est malicieux ou railleur' ou même moqueur (pol. żartobliwy) - 'l'émetteur fait rire le récepteur'. Indépendamment des processus dérivationnels employés par les internautes, les dérivés formés sur les acronymes des noms de partis politiques Prawo i Sprawiedliwość, Platforma Obywatelska ne permettent qu'une interprétation négative.

\section{CONCLUSION}

L'analyse des dérivés simples formés à partir des sigles $P i S$ et $P O$ prouve que la suffixation est un processus au service du discours d'hostilité, bien que les dérivés simples soient moins aptes à manifester l'attitude émotionnelle (l'expressivité via les suffixes) que les dérivés composés ou les contaminations (l'expressivité via les bases et différents affixes).

En fait, à travers la dérivation expressive, les émetteurs manifestent leur aversion, discréditent, stigmatisent, se montrent hostiles envers les référents auxquels renvoient les formes dérivées. Parois cette hostilité n'est pas exprimée ouvertement, par exemple le choix des formes PiSuś ou PiSiątko fait intervenir la dérivation méliorative. Pourtant, même dans ces cas-là, il s'agit de créer des étiquettes, des noms insultants, qui remplacent les formulations neutres. Certains de ces dérivés sont plus fréquents que les autres, mais tous fonctionnent en tant que formes occasionnelles et remplissent les conditions de l'insulte - leur expressivité sert à humilier l'autre, à l'injurier, à lui attribuer des connotations péjoratives. Bien sûr, la suffixation expressive facilite la tâche aux internautes, pourtant l'analyse des dérivés avec les suffixes neutres, classifiés comme non expressifs, comme PiSowiec, POwiec ${ }^{36}$,

${ }^{35} \mathrm{Ce}$ sont les remarques sur l'extension et la sphère de l'emploi typique/usuel du mot (Nagórko, Łaziński, Burhardt, 2004, p. XXXII).

${ }^{36}$ Par ex. : „Pisowiec to jeszcze nie Polak, to małość. Takie są wyniki demoralizatorstwa Kaczyńskiego", fr. «<Pisowiec $>$ n'est pas encore un Polonais, c'est une petitesse. Tel est le résultat de la démo- 
dévoile qu'ils peuvent devenir péjoratifs (jamais mélioratifs), si le contexte le permet. Le discours public des médias électroniques est un témoin des processus dérivationnels qui reflètent l'hostilité présente dans la vie politique polonaise actuelle, hostilité dont l'un des instruments est la création d'insultes bâties sur le nom du parti politique, défini comme ennemi.

ralisation effectuée par Kaczynski » (http://www.tokfm.pl/blogi/off2/2017/03/pisowiec_to_jeszcze_nie_ polak_to_malosc_takie_sa_wyniki_demoralizatorstwa_kaczynskiego/1,) ; „Patrząc na 8 lat rządów Platformy Obywatelskiej możemy stwierdzić, że POwcy to/ - ludzie pozbawieni honoru /- łgarze polityczni i społeczni (...) POwcy to autorzy przemysłu nienawiści... Ktoś ma jeszcze wątpliwości, kim jest POwiec??", fr. « Quand on regarde les huit années du pouvoir de $P O$ on peut constater que [POwcy] ce sont les gens sans honneur, menteurs politiques et sociaux $(\ldots)<$ POwcy $>$ sont les auteurs de l'industrie de haine... Quelqu'un doute encore de l'identité de $<$ Powiec $>$ ? » (http://forum.interia.pl/powiec-kimjest--tematy,dId,2728815). [trad. de l'auteure] 


\section{BIBLIOGRAPHIE}

Bieliński, J. (2013). Niemożliwa tożsamość: wizerunki podmiotów relacji „my-oni”. In X. Bukowska, B. Markowska (éds). To oni sa wszystkiemu winni... Język wrogości w polskim dyskursie publicznym (pp. 75-89). Warszawa : Wydawnictwo Trio.

Bralczyk, J., Mosiołek-Kłosińska, K. (2000). Język w mediach masowych. Warszawa : Rada Języka Polskiego przy Prezydium PAN.

Bralczyk, J., Mosiołek-Kłosińska, K. (2001). Zmiany w publicznych zwyczajach językowych. Warszawa : Rada Języka Polskiego przy Prezydium PAN.

Bukowska, X., Markowska, B. (éds). To oni sq wszystkiemu winni... Język wrogości w polskim dyskursie publicznym. Warszawa : Wydawnictwo Trio.

Burkacka, I. (2012). Kombinatoryka sufiksalna $w$ polskiej derywacji odrzeczownikowej. Warszawa : Uniwersytet Warszawski.

Głowiński, M. (2009). Nowomowa i ciagi dalsze: szkice dawne i nowe. Kraków : Towarzystwo Autorów i Wydawców Prac Naukowych Universitas.

Grabias, S. (1981). O ekspresywności języka: ekspresja a słowotwórstwo. Lublin : Wydawnictwo Uniwersytetu Marii Curie-Skłodowskiej.

Grevisse, M., Goosse, A. (2008). Le bon usage. Quatorzième édition. Bruxelles : Édition De Boeck Univeristé.

Grochowski, M. (1982). Zarys analizy semantycznej grupy jednostek wyrażających negatywne etycznie relacje osobowe (kpina, zniewaga, upokorzenie). Polonica, VIII, pp. 52-72.

Grochowski, M. (1991). Przekleństwo i wulgaryzm jako kwalifikatory pragmatyczne jednostek leksykalnych. Torun : Wydawnictwo Naukowe Uniwersytetu Mikołaja Kopernika.

Grochowski, M. (2002). Stownik polskich przekleństw i wulgaryzmów. Warszawa : Wydawnictwo Naukowe PWN.

Grzegorczykowa, R. (2012). Świat widziany poprzez stowa. Szkice z semantyki leksykalnej. Warszawa : Wydawnictwo Uniwersytetu Warszawskiego.

Grzegorczykowa, R., Puzynina, J. (1979). Stowotwórstwo wspótczesnego języka polskiego: rzeczowniki sufiksalne rodzime. Warszawa : Państwowe Wydawnictwo Naukowe.

Jonasson, K. (1994). Le nom propre. Constructions et interprétations. Louvain-la-Neuve: Duculot. Retrieved from https://doi.org/10.3917/dbu.jonas.1994.01

Kamińska-Szmaj, I. (2006). Apelatywizacja nazw własnych w inwektywach politycznych. In Z. Abramowicz, E. Bogdanowicz (éds), Onimizacja i apelatywizacja (pp. 103-110). Białystok : Wydawnictwo Uniwersytetu w Białymstoku.

Kamińska-Szmaj, I. (2007). Agresja językowa w życiu publicznym: leksykon inwektyw politycznych 1918-2000. Wrocław : Wydawnictwo Uniwersytetu Wrocławskiego.

Kaproń-Charzyńska, I. (2014). Pragmatyczne aspekty słowotwórstwa: funkcja ekspresywna i poetycka. Torun : Wydawnictwo Naukowe Uniwersytetu Mikołaja Kopernika.

Kudra, B. (2001). Kreatywność leksykalna $w$ dyskursie politycznym polskiej prasy lat osiemdziesiąych i dziewięćdziesiatych. Łódź : Wydawnictwo Uniwersytetu Łódzkiego.

Lagorgette, D., Larrivée P. (2004). Introduction. Langue Française, 144, pp. 3-12. Retrieved from https://doi.org/10.3917/lf.144.0003

Markowska, B. (2013). Jacy „my” i jacy „oni”? Analiza semantyczna nazw i etykiet. In X. Bukowska, B. Markowska (éds), To oni sa wszystkiemu winni... Język wrogości w polskim dyskursie publicznym (pp. 19-53). Warszawa : Wydawnictwo Trio.

Nagórko, A. (2003). Zarys gramatyki polskiej. Warszawa : Wydawnictwo Naukowe PWN.

Nagórko, A., Łaziński, M., Burkhardt, H. (2004). Dystynktywny słownik synonimów. Kraków : Universitas. 
O'Keeffe, A. (2011). Media and Discourse Analysis. In J. Gee, M. Handford (éds), The Routledge Handbook of Discourse Analysis (pp. 441-454). London : Routledge. Retrieved from http://hdl. handle.net/10395/1681

Pałuszyńska, E. (2006). Pragmatyczne aspekty użycia nazw własnych w tekstach prasowych. In Z. Abramowicz, E. Bogdanowicz (éds), Onimizacja i apelatywizacja (pp. 165-171). Białystok : Wydawnictwo Uniwersytetu w Białymstoku.

Pisarek, W. (2000). Język w mediach, media w języku. In J. Bralczyk, K. Mosiołek-Kłosińska (éds), Język w mediach masowych (pp. 9-18). Warszawa : Rada Języka Polskiego przy Prezydium PAN.

Pisarkowa, K. (1978). Hasło honor jako przedmiot analizy pragmatycznojęzykowej. Polonica, IV, pp. 117-135.

Sałkowska, M. (2013). Jak „inny” staje się „obcym”, albo kiedy różnica staje się piętnem. In X. Bukowska, B. Markowska (éds), To oni sq wszystkiemu winni... Język wrogości w polskim dyskursie publicznym (pp. 91-115). Warszawa : Wydawnictwo Trio.

Satkiewicz, H. (2000). Językowe przejawy agresji w mediach. In J. Bralczyk, K. Mosiołek-Kłosińska (éds), Język w mediach masowych (pp. 28-33). Warszawa : Rada Języka Polskiego przy Prezydium PAN.

\section{SOURCES INTERNET CONSULTÉES}

Andreth. (2017, May). Retreived from https://www.wykop.pl/wpis/23976337/chyba-nielada-poplochw-zaciszu-biur-mlodziezowek-/

Antonina. (2015, October 17). POwiec - kim jest? Retrieved from http://forum.interia.pl/powiec-kimjest--tematy,dId,2728815

Biblioteka. (2007). PiSiulki dwie, ciemna i jasna, i PiSiulek jeden. Retrieved from http://pl.soc.polityka. narkive.com/HMF1nHFY/pisiulki-dwie-ciemna-i-jasna-i-pisiulek-jeden

Blog lekkoducha. (2014). Retrieved from http://lekko-duch.blog.onet.pl/2014/11/05/pisiaki-tv-trwam-iinne-wiesci-z-zakrystii/

Bolek. (2016, April 8). Paweł Szałamacha: Z obniżką VAT musimy poczekać. Retrieved from http://m.in teria.pl/komentarze, 2315111,4211 ,strona, 7

Gość. (2014, May 10). Została na bruku. Retrieved from http://www.wiescisokolowskie.pl/zostala-nabruku,08I8GHWAWpES3ZOofHK1TCB9bO936V2DSA576wzs325N946TZl.html

Gość. (2015, April, 6). Kamiński z wyrokiem. A co z AFERĄ DUDY? Retrieved from http://forum.echodnia. eu/kaminski-z-wyrokiem-a-co-z-afera-dudy-t290212/

jan_g. (2013, July 5). Elektrim. Retrieved from http://www.bankier.pl/forum/temat_re-12-straconychlat-dla-polski-gdyby-rzadzil-pisiaczek, $12972808 . \mathrm{html}$

Mintor. (2013, February 25). Retrieved from http://www.wiocha.pl/komentarze_uzytkownika,217838,308

Moher. (2013, August 13). „Co z budową Centrum Wystawienniczo-Kongresowego w Jasionce?”. Retrieved from http://supernowosci24.pl/co-z-budowa-centrum-wystawienniczo-kongresowego-wjasionce/

Narciasz. (2016). Discussion: Andrzej Dupa. Retreived from http://soc.culture.polish.narkive.com/Plwf1 NS5/andrzej-dupa

Próbki cukierków (2017). Retreived from http://www.tokfm.pl/blogi/off2/2017/03/pisowiec_to_jeszcze_ nie_polak_to_malosc_takie_sa_wyniki_demoralizatorstwa_kaczynskiego/1

Salon24. (2010). Retrieved from http://stoje-z-boku-i-patrze.salon24.pl/244478,straszna-pisowa- wyrzucona-z-wiadomosci

Salon24. (2013). Retrieved from https://www.salon24.pl/u/zamkinapiasku/490632,byc-polakiem-niejest-latwo-w-polsce 
Strona byłych działaczy Wolnych Związków Zawodowych Wybrzeża. (2013). Retrieved from https://wzzw. wordpress.com/2013/05/31/platfusy-chcecie-zeby-pisory-poslaly-nas-do-mamra-lud-tak- $\% \mathrm{E} 2 \% 98 \% 9 \mathrm{~A} /$

Te-Killa. (2010, July 26). Stenogramy z kabiny pilotów nie będą upublicznione. Retrieved from http://wiadomosci.onet.pl/forum/stenogramy-z-kabiny-pilotow-nie-beda-upublicznione,299257,20, czytaj-popularne.html 